\title{
Analyses of Manufacturing System Matrix Structure for Preven-tive Maintenance using Computer Simulation
}

\author{
Sławomir Kłos ${ }^{1 *}$, Justyna Patalas - Maliszewska ${ }^{1}$ \\ 1 University of Zielona Góra, Faculty of Mechanical Engineering, Licealna 9, 65-417 Zielona Góra, Poland
}

\begin{abstract}
The concept of industry 4.0 includes implementation of preventive and predictive mainte-nance of manufacturing systems. In the paper the matrix structure of manufacturing system is proposed that enables the implementation of production processes even in the event of manu-facturing resource failure. The simulation model of the system is prepared using Tecnomatix Plant Simulation software. The simulation experiments are conducted for different buffers capacities and availability of manufacturing resources. The simulation method enables to ana-lyze the impact of the failure of manufacturing resources on the throughput and average lifespan of products. The matrix structure enables to predict of the manufacturing system per-formance reduction due to partial or total failure of manufacturing resources and evaluate the changes of the work in process level.
\end{abstract}

Keywords: preventive maintenance; computer simulation; throughput; average lifespan of product.

\section{Introduction}

The computer simulation method is usually used for performance analysis of behavior of manufactur-ing systems and study of production processes flow. The computer simulation is especially important in the concept of Industry 4.0 for implementation of a smart factory and building cyber twins. Other side the automation and robotization of production systems requires the implementation of mechanisms of predictive and preventive maintenance. One of the simple but effective method for prevent the manufac-turing systems for total breakdown is using redundant resources. In the paper a simulation model of ma-trix manufacturing system is proposed. For the model simulation experiments are prepared and conducted to analyze the impact of reduction of manufacturing resources availability on the total performance of the system.

Using simulation methods for analysis of manufacturing systems behavior is the subject of many sci-entific publications. Computer simulation has been used for analysis the effectiveness of preventive or corrective maintenance, and improve overall operational reliability, the utilization of manufacturing re-sources, and the productivity of manufacturing systems [1]. Computer simulation offers very effective tools for visualizing, understating, and analyzing the dynamics of manufacturing systems [2]. Fernandes and Carmo-Silva presented a simulation study of the role of sequence-dependent set-up times in decision making at the order release level of a workload controlled make-to-order flow-shop [3]. They indicated that the local strategy, which has been traditionally adopted in practice and in most of the studies dealing with sequence-dependent set-up times, does not always give the best results. Kłos and Patalas - Maliszewska studied the impact of buffer allocation on the manufacturing system performance using the computer simulation method 
[4] and Kłos and Trebuna analyzed the impact of buffer and allocation of workers on the throughput and average lifespan of products in discrete manufacturing system [5]. Fraz-zon et al. propose a simulation model to evaluate the improvements in performance provided by integrat-ed intelligent maintenance systems and spare-part supply chains [6]. Wan et al. analysed the issue of ac-tive preventive maintenance and the related system architecture for manufacturing big data solutions in smart manufacturing systems [7]. The data collection and data processing stages with respect to the Cloud computing environment were presented. $\mathrm{Ni}$ and Jin presented new decision support tools based on math-ematical algorithms and simulation tools for effective maintenance operations [8]. A comprehensive overview over recent simulation applications in manufacturing and business is provided by Jahangirian et al. [9]. The matrix structures of manufacturing systems are described by Greschke et al. [10]. Lu and Zou proposes a novel opportunistic preventive maintenance scheduling methodology for the serial-parallel multistage manufacturing systems, where PM is performed to improve the reliability and quality of a product [11].

In the paper the simulation model of matrix (parallel serial, multistage) manufacturing is proposed. The system includes manufacturing resources and buffers. The material flow is controlled using dispatching rules allocated on the manufacturing resources (exit strategy of products from resources). The manufac-turing routes not includes reversions what guarantee that the manufacturing system I deadlock- and star-vation-free. The research problem is formulated as follows:

Given is parallel serial manufacturing system (matrix topology) that includes determined number of man-ufacturing resources and buffers. The processing times and production program are defined. The materi-al flow is controlled by dispatching rules allocated on resources and buffers. What is the impact of changing of availability of manufacturing resources on the throughput and average lifespan of products in the system for different allocation of buffer capacities.

The proposed model of manufacturing system is prepared using Tecnomatix Plant Simulation software. The description of the model and the results of experiments are presented in the next chapter.

\section{Methods}

The model includes 6 resources divided into 2 technological stages. First stage includes resources
M11, M21, M31 and the second stage resources M21, M22, M23. Between each two resources from different stages a buffer is allocated. The structure of the manufacturing system is presented in the Fig. 1.

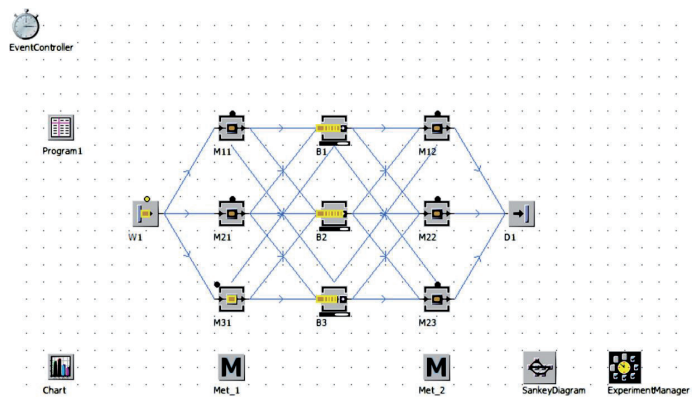

Fig. 1: The model of manufacturing system in Tecnomatix Plant Simulation.

The all resources (machines) $M_{11}, M_{21}, M_{31}$ are connected with buffers $B_{1}, B_{2}$, and $B_{3}$ and similarly the buffers are connected with all machines $M_{12}, M_{22}$, $\mathrm{M}_{23}$. The material flow is controlled using dispatching rules allocated on resources and buffers. On the resources $M_{11}, M_{21}, M_{31}$ is allocated dispatching rule that select the successor with minimum number of products in a buffer. On the buffers $B_{1}, B_{2}, B_{3}$ is allocated dispatching rule that select the resources for product with minimum processing time. In the system five different products is processed $E_{1}$, $E_{2}, \ldots E_{5}$. The structure of the system guarantee that each route in-cludes both two stages of the manufacturing process (for example $M_{11}->B_{1}->$ $M_{12} ; M_{11}->B_{2}->M_{22} ; M_{11}->B_{3}->M_{23}$; etc.).

The processing times are determined using lognormal distribution. The lognormal distribution is a contin-uous distribution in which a random number has a natural logarithm that corresponds to a normal dis-tribution. The realisations are nonnegative, real numbers. The density of the lognormal distribution Lognor $(\sigma, \mu)$ is calculated as follows [12]:

$$
f(x)=\frac{1}{\sigma_{0} x \sqrt{2 \pi}} \cdot \exp \left[\frac{-\ln \left(x-\mu_{0}\right)^{2}}{2 \sigma_{0}^{2}}\right]
$$

where $\sigma$ and $\mu$ are respectively mean and standard deviations and are defined as follows:

$$
\mu=\exp \left[\mu_{0}+\frac{\sigma_{0}}{2}\right]
$$

$$
\sigma^{2}=\exp \left(2 \mu_{0}+\sigma_{0}^{2}\right) \cdot\left(\exp \left(\sigma_{0}^{2}\right)-1\right)
$$


The maximum of the density function is defined as:

$$
\exp \left(\mu_{0}-\sigma_{0}^{2}\right)
$$

The operation times for all products are presented in the Table 1. The operation times are defined in seconds for stage 1 - machines $M_{11}, M_{21}, M_{31}$ and stage 2 - machines $M_{21}, M_{22}, M_{23}$.

Table 1: The processing times of the products.

\begin{tabular}{|l|l|l|l|l|}
\hline Product & Stage 1 & \multicolumn{3}{|c|}{ Stage 2 } \\
\hline & $\sigma^{2}$ & $\mu$ & $\sigma^{2}$ & $\mu$ \\
\hline E1 & 18 & 0.1 & 36 & 0.1 \\
\hline E2 & 24 & 0.1 & 24 & 0.1 \\
\hline E3 & 30 & 0.1 & 30 & 0.1 \\
\hline E4 & 36 & 0.1 & 24 & 0.1 \\
\hline E5 & 48 & 0.1 & 36 & 0.1 \\
\hline
\end{tabular}

Table 2: The allocation of buffer capacities as input values of simulation experiments.

\begin{tabular}{|l|l|l|l|l|}
\hline Experiment № & B1 & B2 & B3 & E \\
\hline Exp 01 & 1 & 1 & 1 & 3 \\
\hline Exp 02 & 1 & 1 & 6 & 8 \\
\hline Exp03 & 5 & 2 & 2 & 9 \\
\hline Exp04 & 10 & 1 & 1 & 12 \\
\hline Exp 05 & 1 & 1 & 10 & 12 \\
\hline Exp 06 & 4 & 5 & 4 & 13 \\
\hline Exp 07 & 1 & 9 & 4 & 14 \\
\hline Exp 08 & 2 & 9 & 7 & 18 \\
\hline Exp09 & 9 & 4 & 6 & 19 \\
\hline Exp 10 & 10 & 3 & 8 & 21 \\
\hline Exp 11 & 9 & 8 & 9 & 26 \\
\hline Exp 12 & 10 & 10 & 10 & 30 \\
\hline
\end{tabular}

The simulation experiments are conducted for different allocation of buffer capacities. The allocation of buffer capacities for 12 simulation experiments are presented in the Table 2. The buffers capacity is determined using random number generator of Tecnomatix Plant Simulation. The range of capacities could change from 1 to 10 and in the first step 60 random variants of buffer capacities is generated. In the last column of the Table 1 , the sum of allocated buffer capacity is presented. The output values of the simulation experiments are selected throughput per hour and average lifespan of products. The values of throughput were ordered in ascending order and next every fifth experiment was selected to capture significant changes in the manufacturing system performance. The availability of all manufacturing re-sources on the beginning are defined as 95\% and the time of each simulation experiments is determined as $8 \mathrm{~h}$. For each simulation experiments 3 observations are realized. The simulation experiments are re-peated for different availability of manufacturing resources and the impact on the throughput and average lifespan of product is observed. The results of simulation experiments are presented and discussed in the next chapter.

\section{The results of simulation experiments results}

The simulation experiments are prepared for 5 different variants of the resources availability. An example of different resources availability is presented in the Fig. 2, where $M_{21}=M_{22}=90 \%$ and the rest of resources availability is $95 \%$.

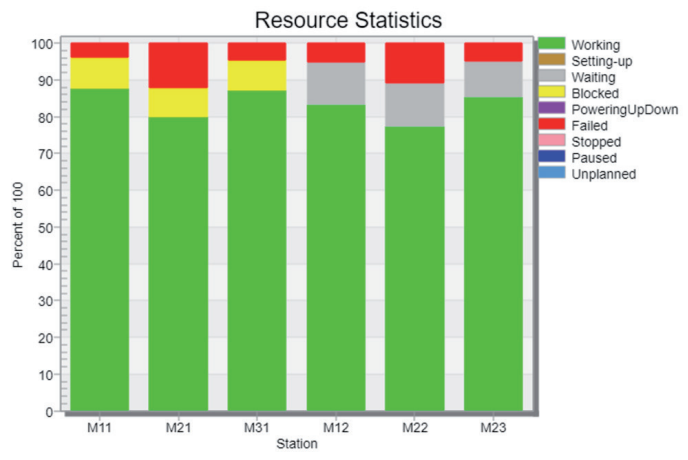

Fig. 2: The example of resources statistics where availability is $M_{11}=$ $M_{31}=M_{12}=M_{23}=95 \%$ and $M_{21}=M_{22}=90 \%$.

First the simulation experiments are conducted for different availabilities of manufacturing resources. In the Fig. 3 the results of experiments where the availability of resources was reduced about $5 \%$. The great-est value of throughput ( $T h=327$ products per hour) is reached for the system where all resources availa-bility is $95 \%$ and for the maximum capacity of buffers (Exp $\left.12 \mathrm{~B}_{1}=\mathrm{B}_{2}=\mathrm{B}_{3}=10\right)$.

From the charts presented in the Fig. 3 results that for the throughput of the system is important which resource availability is reduced. Generally, the greatest impact on the reduction of the throughput of the system has the availability of resource $M_{21}$. The proper allocation of the buffer 


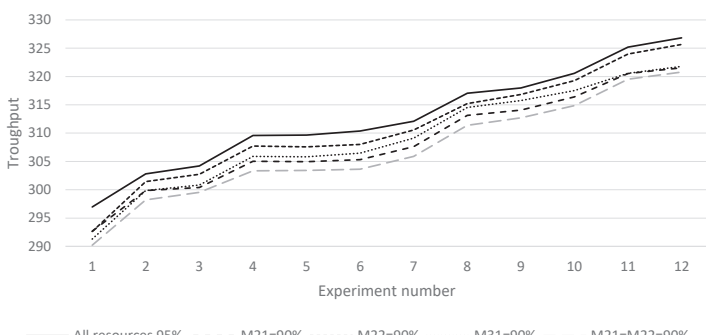

Fig. 3: The throughput of the system for availability of resources $95 \%$ and $90 \%$.

capacity (increasing the buffer capacities) enables to improve the throughput for each variants of availability of manufacturing resources. For example, the throughput $T h=310$ products per hour could be achieved for the Exp 06 for all resources with availability of $95 \%$ and $\mathrm{Th}=311$ ich reached for Exp 08 where availability of resources $M_{12}=M_{22}=90 \%$. It means that the proper allocation of buffer capacity enables to reduce impact of low availability of resources. Increasing of buffers ca-pacity result with increasing a level of work in process. The Fig. 4 presents the average lifespan of products for the simulation experiments.

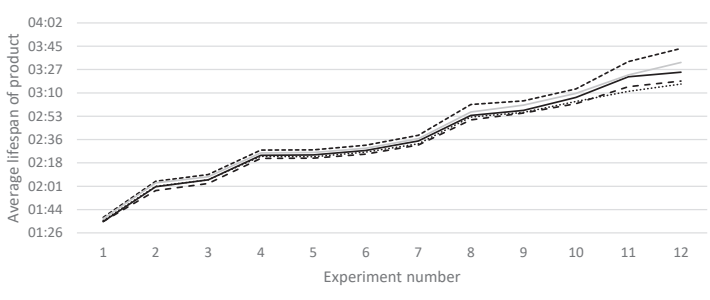

All resources 95\% - - - M21=90\% --- M22=90\% ……..... M31=90\% - M21=M22=90\%

Fig. 4: The average lifespan of products of availability of resources $95 \%$ and $90 \%$.

The products stay in the system from $L f=1: 35$ for Exp 01 to $L f=3: 34$ for Exp 12, depend from the allocated buffer capacity. The differences for individual experiments are rather small, for example for the variant where availability $M_{22}=90 \%$ for Exp $12 \mathrm{Lf}=3: 19$ and for the same experiment and variant where availability of all resources is $95 \%$ $\mathrm{Lf}=3: 25$ (difference 6 seconds). The next simulation experiments are conducted for cases when the manufacturing resources are failure (availability 1\%). In the Fig. 5 the system throughput where individual resources availability is $1 \%$ is presented.

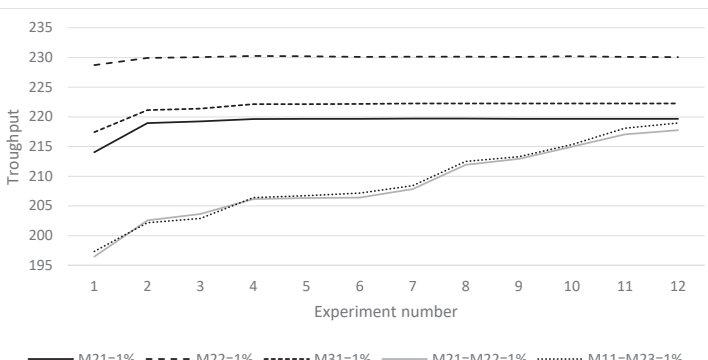

Fig. 5: The throughput of the system for resources availability $1 \%$.

The analysis of the system behavior in a case of the failures of individual machine shows that if the availability of resource is reduced to $1 \%$ the allocation of buffer capacities has no impact on the throughput. If the availability of machine $\mathrm{M}_{22}=1 \%$ the value of throughput is constant and for ma-jority variants of buffer capacity allocation is constant $\mathrm{Th}=230$, and similar for availability of $\mathrm{M}_{31}=1 \%$ the throughput is $T h=220$ and for $M_{21}=1 \%$ Th=220. If two machines are failure in the same time (for example availability of machines $\mathrm{M}_{11}=\mathrm{M}_{23}=1 \%$ ) the throughput depends on the buffer capacities. The impact of buffer capacity on the average lifespan of products for resources availa-bility $1 \%$ is presented in Fig. 6.

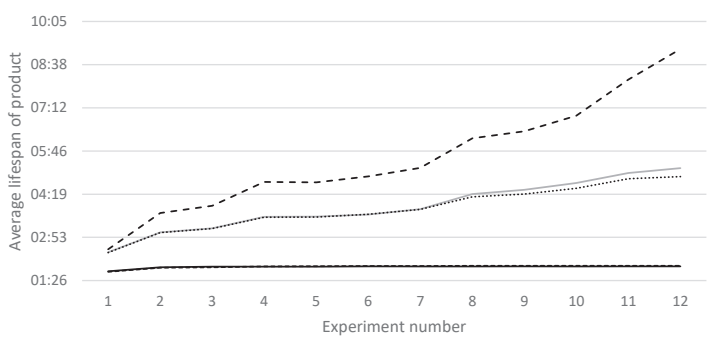

Fig. 6: The average lifespan of products of the system for resources availability $1 \%$.

The level of work in process (average lifespan of products) depends which machine is failure. For ex-ample, if availability of machines $M_{21}=1 \%$ or $M_{31}=1 \%$ then the lifespan is $L f=1: 56$. If the machine $\mathrm{M}_{22}=1 \%$ the average lifespan depends on the allocation of buffer capacity and belongs to the range $\langle 2: 29 ; 9: 10\rangle$. In the case of failure of two machines together (for example $\mathrm{M}_{11}=\mathrm{M}_{23}=1 \%$ ) then the average lifespan of products belongs into the range $\langle 2: 22 ; 5: 11>$. On the base of the analyses of results of simula-tion experiments the conclusions 
and directions of further research are prepared.

\section{Conclusions}

In the papera model of parallel serial manufacturing system is prepared and analyzed using computer simulation. The matrix manufacturing systems thanks redundant manufacturing resources are breakdown resistant what is very important to realize concept of preventive maintenance in Industry 4.0. To guaran-tee the high performance of the system the proper allocation of buffer capacity is required. The following conclusion results from the analysis of the simulation experiments could be formulated:

$\checkmark$ the allocation of buffer capacity has significant impact on the throughput of the system - proper buffer allocation enables to increase throughput of the system about 10\%,

$\checkmark$ the reduction the availability of a manufacturing resource about $5 \%$ results with reduction of the system throughput about $1 \%$,

$\checkmark$ the reduction of availability of individual manufacturing resources has the same impact on the average; lifespan of products,

$\checkmark$ the breakdown of machines for the maximum buffer capacity allocation results with reduction of the throughput of the whole system about 33\% (no matter if one or two machines are failure together),

$\checkmark$ for breakdown of one machine the allocation of buffer capacity has no impact on the through-put of the system.

In the further research the intelligent algorithms based on the artificial neural networks will be created to estimate the probability of machines breakdown for predictive maintenance concept.

\section{Acknowledgments}

This work is supported by program of the Polish Minister of Science and Higher Education under the name "Regional Initiative of Excellence" in 2019 - 2022, project no. 003/RID/2018/19, funding amount 11936596.10 PLN.

\section{References and Notes}

[1] Negahban A., Smith J. S., 2014, Simulation for manufacturing-system design and operation: Litera-ture review and analysis, Journal of Manufacturing Systems, Vol. 33, 2014, pp.241-261.

[2] Seleima A., Azaba A., AlGeddawy T., 2012, Simulation Methods for Changeable Manufacturing, 45th CIRP Conference on Manufacturing Systems 2012, Procedia CIRP 3, pp. 179 $-184$.

[3] Fernandes N. O., Carmo-Silva S., 2011, Order release in a workload controlled flow-shop with se-quence-dependent set-up times, International Journal of Production Research, Vol. 49(8), pp. 2443-54.
[4] Kłos S., Patalas-Maliszewska J., An Approach to Buffer Allocation, in Parallel-Serial Manufacturing Systems Using the Simulation Method, 2018, Springer International Publishing, 2018 -- Advances in Intelligent Systems and Computing, vol. 747, s. 225 - 235.

[5] Kłos S., Trebuna P., 2015, Using computer simulation method to improve throughput of production systems by buffers and workers allocation, Management and Production Engineering Review, Vol. 6, pp. 60-69.

[6] Frazzon E. M., Silva L. S., Pires M. C., 2016, Simulation-based performance evaluation of a concept for integrating intelligent maintenance systems and spare parts supply chains, IFAC-PapersOnLine, Vol. 49-12, pp. 1074-1079.

[7] Wan, J., Tang, S., Li, D., Wang, S., Liu, C., Abbas, H., Vasilakos, A.V., 2017, A manufacturing big data solution for active preventive maintenance, IEEE Transactions on Industrial Informatics, Vol. 13, pp. 2039 - 2047.

[8] Ni J, Jin X, 2012, Decision Support Systems for Effective Maintenance, Operations, CIRP Annals - Manufacturing Technology, Vol. 61, pp. 411-414.

[9] Jahangirian M, Eldabi T, Naseer A, Stergioulas LK, Young T. 2010, Simulation inmanufacturing and business: a review, European Journal of Operational Research, Vol. 203(1), pp. $1-13$

[10] Greschke P, Schönemann M, Thiede S, Herrmann C. 2014, Matrix structures for high volumes and flexibility in production systems, Procedia CIRP 2014;17, pp. 160-5.

[11] Lu B., Zhou X., Opportunistic preventive maintenance scheduling for serial-parallel multistage manufacturing systems with multiple streams of deterioration, 2017, Reliability Engineering and System Safety Vol. 168, pp. 116-127.

[12] Tecnomatix Plant Simulation v. 14.02 - online documentation. 